\title{
RISK MEASURES AND EFFICIENT USE OF CAPITAL ${ }^{1}$
}

\author{
BY
}

\section{Philippe Artzner ${ }^{2}$, Freddy Delbaen ${ }^{3}$ and Pablo Koch-Medina ${ }^{4}$}

\begin{abstract}
This paper is concerned with clarifying the link between risk measurement and capital efficiency. For this purpose we introduce risk measurement as the minimum cost of making a position acceptable by adding an optimal combination of multiple eligible assets. Under certain assumptions, it is shown that these risk measures have properties similar to those of coherent risk measures. The motivation for this paper was the study of a multi-currency setting where it is natural to use simultaneously a domestic and a foreign asset as investment vehicles to inject the capital necessary to make an unacceptable position acceptable. We also study what happens when one changes the unit of account from domestic to foreign currency and are led to the notion of compatibility of risk measures. In addition, we aim to clarify terminology in the field.
\end{abstract}

\section{KEYWORDS}

Acceptance set, accounting, currency compatibility, eligible asset, no acceptability arbitrage, non acceptability of leverage, numéraire, risk-free investment, risk measurement, supervision.

\section{INTRODUCTION}

Measuring the risk of a portfolio of assets and liabilities by determining the minimum amount of supporting capital that needs to be added to the portfolio to make the future value "acceptable" has now become a standard in the

\footnotetext{
1 This paper elaborates, with a major change, on presentations to the Isaac Newton Institute, DQF Program, February 2005, the Solvency II Tagung, University of Karlsruhe, April 2005, the ASTIN/ AFIR Colloquium, Zürich, September 2005 and to the DGVFM Insurance Day, Cologne, April 2006. Thanks are due to referees for insisting on the need of comments related to the setting of the paper in the current literature, and to Jean-Marc Eber and Patrice Poncet for interesting discussions.

2 Partial supports from AERF/CKER, The Actuarial Foundation and from the Isaac Newton Institute are gratefully acknowledged.

3 Partial support from Credit Suisse is gratefully acknowledged.

4 This author expresses his personal view in the paper.
} 
financial services industry. The theoretical framework is that of coherent risk measures at first described in Artzner et al. (1997 and 1999).

In those papers the basic approach consisted in defining a set of future values of financial positions viewed as acceptable (the acceptance set), specifying a traded asset in which the supporting capital may be invested (the "eligible" asset, which is often chosen to be the "risk-free" asset in the given currency), and determining the minimum investment in the eligible asset that needs to be added to the original portfolio to make it acceptable (the "required" capital). This minimum investment defines a risk measure which is coherent if the acceptance set is well chosen.

The minimum required capital will of course depend on the definition of acceptability but also on the choice of the eligible asset. Given the acceptance set, the limitation to a single eligible asset does not necessarily yield the least amount of required capital (extra cash at the initial date required to turn an unacceptable position into an acceptable one). This is particularly evident when assets and liabilities are denominated in both domestic and foreign currency. Intuitively, the supporting capital for such a portfolio should be invested in a mixture of assets in the various currencies rather than in a single chosen currency. As illustrated by an example in the Appendix, if one allows for supporting capital to be invested in the "risk-free" assets of both currencies - rather than only in one of them - less capital is ultimately required to make a non-acceptable portfolio acceptable. The focus of this paper is therefore on investigating what happens when one allows multiple eligible assets to be used.

We next discuss the structure of the paper. Comments on and links to the current literature are found in Sections 1,2 and 3.

Section 1 provides a review of "single-eligible-asset" coherent risk measures and then characterizes when two such risk measures coincide. This is applied to the question of whether it is possible to choose an optimal eligible asset in the sense that any alternative choice of an eligible asset leads to higher capital requirements. We take the opportunity to clarify, in Remarks 1 to 5 , several topics of general interest such as:

(i) the conceptual primacy of the acceptance set in risk measurement as introduced in Artzner et al. (1999),

(ii) the relativity of the notion of a "risk-free" investment,

(iii) the advantages of using cash as a unit of account from an interpretational perspective,

(iv) the different uses of the term "numéraire" in economics or business and mathematical finance, and the distinction of the potential uses of the eligible asset as an investment vehicle and as a unit of account,

(v) the distinction between the supervisory and utility approaches to risk measurement.

Section 2, the main section of the paper, investigates the use of multiple eligible assets. We look at the minimum investment in a portfolio of a given set of 
eligible assets that needs to be added to the original portfolio to make it acceptable. It is not clear from the outset that defining such a multiple-eligible-asset risk measure will yield a risk measure with "reasonable" properties. However it can be shown, under a condition that we call "non acceptability-arbitrage", that the original risk measure is identical to a coherent risk measure defined with anyone of the original assets acting as the single eligible asset and with the new acceptance set obtained from the original acceptance set by augmenting it by the future values of portfolios of eligible assets which have initial value zero. The original multi-eligible-asset risk measure therefore satisfies the usual properties of a single-eligible-asset coherent risk measure: monotonicity, positive homogeneity, subadditivity and translation invariance w.r.t. anyone of the eligible assets. It also satisfies a generalized translation invariance property.

Section 3 tackles another issue which arises naturally in a multi-currency seting: what happens when we change the currency we account in? A natural requirement is that if a company were to calculate risk using a domestic or a foreign risk measure, these measures should be compatible in the sense that it should not matter whether we first calculate the capital requirement when expressing the future value of a position in the foreign currency and then translate said capital requirement into domestic currency at the spot rate (exchange rate at time $t=0$ ), or perform all calculations in domestic currency in the first place. An interesting observation is that if a domestic risk measure and a foreign risk measure are defined via expected shortfall (i.e. TailVaR) in the respective currencies, they will generally fail to be compatible.

\section{REACHing THE ACCEPTANCE SET With A Single ELIGIBLE ASSET: INVESTING VERSUS ACCOUNTING}

Coherent one-period time $t=0$ measurement of the risk of the time $t=1$ value of financial positions is best approached by taking two sets of primitive objects. First we have

(i) a probability space $(\Omega, \mathcal{F}, \mathbb{P})$ assumed finite, for simplicity, representing the possible states of nature at time $t=1$,

(ii) the space $L^{0}=L(\Omega, \mathcal{F})$ of measurable functions on $(\Omega, \mathcal{F})$ representing the possible time $t=1$ values of financial positions that can be entered by a risk taker,

(iii) an "acceptance set" $\mathcal{A}$, a set of random variables on $(\Omega, \mathcal{F}, \mathbb{P})$, representing the future values of financial positions that are in line with the risk tolerance of the supervisor.

The set $\mathcal{A}$ describes the future value at time $t=1$ of financial positions expressed in nominal terms, i.e. in time $t=1$ money, declared acceptable by the supervisor at time $t=0$. Here, supervisor is a generic term used to denote any stakeholder who has reason to impose a risk constraint on the financial positions 
entered by the risk taker, who is committed to stay within these constraints. Examples of such a stakeholder could be an insurance supervisor or the board of a company. Notice that $\mathcal{A}$ may depend on $\mathbb{P}$, for instance reflecting some tolerance of the supervisor for low probability losses. The acceptance set does not imply any preferences of the supervisor except the obvious one implied by the division of the universe of future values of financial positions into acceptable and unacceptable ones.

Then a fourth object is introduced, which satisfy the following definition:

Definition 1. An eligible asset $r$ is a traded instrument exchanging one unit of time 0 money for a strictly positive number $r(\omega)$ of time 1 money, where $\omega$ is the state of nature at time 1.

(iv) a random variable $r$ on $(\Omega, \mathcal{F}, \mathbb{P})$ representing the future values of an eligible asset. It will be used by the risk manager to align unacceptable positions with the supervisor's risk tolerance.

The eligible asset represented by $r$ is chosen by the risk manager to change unacceptable positions into acceptable ones by adding a capital injection (time 0 money) invested into this asset. This risk management action comes at a cost and the purpose of a risk measure - as initiated in Artzner et al. (1997 and 1999) and extended in this paper - is to identify the minimum cost at which acceptability can be achieved. Notice that the choice of eligible asset will imply that a mitigation of the risk associated with that asset is integrated in the risk measurement. The link between amount of required capital and quality of the eligible asset shows up in the following quote where "adequate" may be substitute for "acceptable": "The overall objective of prudential regulation must be to ensure that an insurer maintains, at all times, financial resources which are adequate, both as to amount and quality to ensure there is no significant risk that the liabilities cannot be met as they fall due" (see Committee of European Insurance and Occupational Pensions Supervisors (2007), Section 2.1).

Together with the eligible asset represented by $r$ the acceptance set $\mathcal{A}$ defines the risk measure $\rho_{A, r}$, a functional on the space $L^{0}$, as follows:

$$
\rho_{\mathfrak{A}, r}(X)=\inf \{m \mid X+m r \in \mathcal{A}\} .
$$

The risk measure $\rho_{\mathfrak{A}, r}(X)$ of the future value $X$ of a financial position is therefore the (possibly negatively infinite) infimum of the amounts $m$ of units of time 0 money which, invested at time 0 in the eligible asset represented by $r$ and "added" to the future value $X$ make the modified future value $X+m r$ acceptable.

Remark 1. The conceptual primacy of the acceptance set over the risk measure merits some comment. As emphasized in the "heavy" notation $\rho_{\Re, r}$ the eligible asset represents a choice. Since we are interested in the interpretation of the risk measure as the least amount of additional capital required to "support" an unacceptable position, it is worthwhile to ask whether a different choice of the eligible asset - an asset of a different "quality" - would have led to a more 
efficient capital support - a lesser "amount" of required capital. This question happens to be linked to our desire to extend the theory to a multi-eligibleasset setting.

Remark 2. Note that Artzner et al. (1999) did not require that the eligible asset $r$ be risk-free as is sometimes misquoted. This is important because the notion of risk-free is a relative notion as highlighted for instance in a multi-currency setting where it is not clear whether the domestic or the foreign "risk-free" instrument should be chosen.

Remark 3. As in Artzner et al. (1999), future values of financial positions are expressed in nominal terms, i.e. in time $t=1$ money. In particular we do not use the eligible asset as a numéraire, i.e. as a unit of account (as it is the case in the earlier version of this paper, Artzner, Delbaen and Koch (2005) and in the multi-period treatment in Artzner et al. (2007)). We do not lose generality by doing so and by expressing values in nominal terms we will avoid confusion when dealing with several eligible assets. We use the word nominal in its traditional meaning: nominal "dollars" defined (in the glossary to Stickney, Weil and Davidson (1991)) as "measuring unit giving no consideration to differences in the general purchasing power of the dollar over time". Note that this is the language of industry participants and observers referring to cash, "legal tender of all debts, public or private".

Remark 4. The term "numéraire" is often used to denote what we have called an eligible asset, a vehicle to invest the additional capital required to turn an unacceptable financial position into an acceptable one. We prefer to use "eligible asset" for several reasons:

- there is a risk of confusion since the the term "numéraire" is then being used for both a unit of account and an eligible investment vehicle. For example, it is useful to distinguish Filipovic's (2008) quest for an "optimal numéraire", which is from a unit of account viewpoint, from our quest for an "optimal eligible asset" (see Proposition 1-c at the end of this section).

- one of the benefits of expressing values in terms of a numéraire is that sometimes the right choice of a numéraire leads to more elegant mathematical expressions and enables the use of a particular mathematical machinery. In the present context the main advantage of using the eligible asset (the investment instrument) as a numéraire is that it leads to a slightly simpler version of translation invariance. However, there is the potential pitfall of forgetting the criticality of the choice of the eligible asset as the instrument in which capital injections may be invested. In addition, when we allow for multiple eligible assets there is no natural way in which to choose one of them as a numéraire and any such choice would not lead to a simplification of notation.

Remark 5. The interpretation of the acceptance set as describing the risk tolerance of the supervisor shows that our approach is not concerned with finding an "objective" measure of risk such as in Aumann and Serrano (2006). This 
interpretation also highlights the differences of our approach to a utility like use of risk measures as in Barrieu and El Karoui $(2002,2005)$ and (partly) Föllmer and Schied (2004).

Remark 6. We do not require here acceptance cones to be closed hence $X+$ $\rho_{\mathfrak{A}, r}(X) r$ which has zero risk measure is not necessarily in $\mathcal{A}$. We note that $\rho_{\mathfrak{A}, r}=$ $\rho_{\bar{A}, r}$ and also that the set where $\rho_{\mathscr{A}, r}$ is negative coincides with the closure of $\mathcal{A}$.

When the acceptance set $\mathcal{A}$ satisfies some "coherence" requirements, namely to be a convex cone of $L^{0}$ with vertex at the origin, containing $L_{+}^{0}$ and not intersecting the interior of $L_{-}^{0}$, the risk measure associated to an eligible asset $r$ is coherent, i.e. satisfies the four conditions:

- monotonicity: for each couple $(X, Y)$, if $X \geq Y$ then $\rho_{\mathfrak{A}, r}(X) \leq \rho_{\mathfrak{A}, r}(Y)$,

- translation invariance: for each constant $a$ and each $X, \rho_{\Re, r}(X+a r)=\rho_{\Re, r}(X)-a$,

- positive homogeneity: if $\lambda \geq 0$ then for each $X, \rho_{\Re, r}(\lambda X)=\lambda \rho_{\mathcal{A}, r}(X)$,

- subadditivity: for each couple $(X, Y), \rho_{\mathfrak{A}, r}(X+Y) \leq \rho_{\mathfrak{A}, r}(X)+\rho_{\mathfrak{A}, r}(Y)$.

An interesting problem is the question of when $\rho_{\mathscr{A}, r}=\rho_{\mathfrak{B}, S}$ for given acceptance sets $\mathcal{A}$ and $\mathcal{B}$ and eligible assets $r$ and $s$.

Proposition 1-a. Given coherent acceptance sets $\mathcal{A}$ and $\mathcal{B}$ and eligible assets $r$ and $s$, then $\rho_{\mathcal{A}, r}=\rho_{\mathcal{B}, s}$ holds if and only if the closures of $\mathcal{A}$ and $\mathcal{B}$ coincide and contain both $r-s$ and $s-r$.

Proof. Assume equality of the risk measures. From Remark 6 above we see that the closures of $\mathcal{A}$ and $\mathcal{B}$ must coincide. Further, we find that $\rho_{\mathcal{A}, r}(-s)=$ $\rho_{\Re, s}(-s)=1$ and $\rho_{\mathfrak{A}, s}(r)=\rho_{\Re, r}(r)=-1$ out of which follows by subadditivity of $\rho_{\mathcal{A}, r}$ that the leveraged position $s-r$ belongs to the closure of $\mathcal{A}$ as well as the position $r-s$.

Assume that the closures of $\mathcal{A}$ and $\mathcal{B}$ coincide and that both $r-s$ and $s-r$ belong to the closure of $\mathcal{A}$. For any $X$ and $m$ with $X+m r \in \mathcal{A}$ we have that $X$ $+m s=X+m r+m(s-r)$ belongs to the closure of $\mathcal{A}$. This implies that $\rho_{\mathcal{A}, s} \leq$ $\rho_{\mathfrak{A}, r}$. A similar argument yields $\rho_{\mathfrak{A}, r} \leq \rho_{\mathfrak{A}, s}$ and, thus, $\rho_{\mathfrak{A}, r}=\rho_{\mathfrak{A}, s}$.

To sharpen the result above we will sometimes impose an additional assumption on acceptance sets and their interplay with financial markets:

Assumption $N A L(\mathcal{A})$. Non acceptability of leverage: non-trivial, zero-cost portfolios of eligible assets do not belong to the closure of $\mathcal{A}$.

Remark 1. Portfolios of eligible assets having zero value at time $t=0$ correspond to highly leveraged positions, i.e. long positions in some assets are financed by "borrowed" money obtained by entering short positions in other assets. A "lender" would never accept to lend money in this way if the "borrower" did not have additional capital supporting the position, thus reducing the probability of default of the borrower. Hence, Assumption $N A L(\mathcal{A})$ is a 
rather natural supervisory requirement. For a related, weaker, condition see Section 4.3 in Artzner et al. (1999) and Chapter 7 in Delbaen (2000).

Remark 2. An arbitrage portfolio would have zero-cost and belong to $\mathbb{R}_{+}^{\Omega} \backslash\{0\}$ violating assumption $N A L(\mathcal{A})$.

Proposition 1-b. Given coherent acceptance sets $\mathcal{A}$ and $\mathcal{B}$ such that either $N A L(\mathcal{A})$ or $N A L(B)$ holds and two eligible assets $r$ and $s$, the equality $\rho_{\mathcal{A}, r}=\rho_{\mathcal{B}, s}$ holds if and only the closures of $\mathcal{A}$ and $\mathcal{B}$ coincide and $r=s$.

Proof. Assume equality of the risk measures. From Proposition 1-a above we know that the closures of $\mathcal{A}$ and $\mathcal{B}$ must coincide and that the leveraged positions $s-r$ and $r-s$ belong to the closure of $\mathcal{A}$. If $N A L(\mathcal{A})$ holds then $s-r$ must be the trivial leveraged position, i.e. $r=s$.

We now turn back to the "optimal eligible asset" problem (see Remark 1 and Remark 4 above) which was inspired by the related "optimal numéraire problem" treated in Filipovic (2008). We therefore ask whether, given an acceptance set $\mathcal{A}$ and two eligible assets $r$ and $s$, the functional inequality $\rho_{\mathcal{A}, r} \leq \rho_{\mathcal{A}, s}$ can hold in non-trivial cases.

Proposition 1-c. Given the coherent acceptance set $\mathcal{A}$ and the eligible assets $r$ and $s$ the inequality $\rho_{\mathfrak{A}, r} \leq \rho_{\mathfrak{A}, s}$ is equivalent to the equality $\rho_{\mathfrak{A}, r}=\rho_{\mathfrak{A}, s}$ together with the fact that both $r-s$ and $s-r$ belong to the closure of $\mathcal{A}$. If we assume that $N A L(\mathcal{A})$ holds, then the inequality $\rho_{\mathfrak{A}, r} \leq \rho_{\mathfrak{A}, s}$ is equivalent to $r=s$.

Proof. From the inequality $\rho_{\mathcal{A}, r} \leq \rho_{\mathfrak{A}, s}$ follows that $\rho_{\mathfrak{A}, r}(s) \leq \rho_{\mathfrak{A}, s}(s)=-1$ which means that $s-r$ belongs to the closure of $\mathcal{A}$ as well as $\rho_{\mathfrak{A}, r}(-s) \leq \rho_{\mathfrak{A}, s}(-s)=1$ which means that $r-s$ belongs to the closure of $\mathcal{A}$. The "if" part in Proposition 1-a ensures that $\rho_{\mathfrak{A}, r}=\rho_{\mathfrak{A}, s}$. Under $N A L(\mathcal{A})$ we moreover get $r=s$.

Remark. The corollary above shows that it is not possible to find an eligible asset which provides a capital requirement which is lower than that obtained by using any alternative eligible asset.

\section{REACHING THE ACCEPTANCE SET WITH SEVERAL ELIGIBLE ASSETS: INVESTING}

Consider reaching acceptability by injecting additional capital invested in a larger set of "eligible" assets than just $\{r\}$. Suppose we are given a set $S$ of eligible assets. Denote by $\mathcal{M}(S)$ the linear subspace of $L^{0}$ consisting of future values, in time $t=1$ money, of portfolios of eligible assets in $S$ and denote by $\mathcal{M}_{0}(\mathcal{S})$ the subspace of elements in $\mathcal{M}(S)$ with initial price 0 . Finally, given the time $t=1$ value of a portfolio of traded assets $Z$, we denote by $\pi(Z)$ its price at time $t=0$. 
Definition 2. Multi-eligible-asset risk measure: Given a coherent acceptance set $\mathcal{A}$ and $a$ set $\mathcal{S}$ of eligible assets, the risk measure $\rho_{\mathcal{A}, S}$ is defined by the relation

$$
\rho_{\mathfrak{A}, S}(X)=\inf \{m \mid X+Z \in \mathcal{A}, \text { for } Z \in \mathcal{M}(S) \text { with } \pi(Z)=m\} .
$$

Remark 1. We have generalized the construction of $\rho_{\mathfrak{A}, r}$ since, by definition, this measure is $\rho_{\mathfrak{A},\{r\}}$.

Remark 2. In their Definition 3.1 Frittelli and Scandolo (2006) also introduce general capital requirements using portfolios of elements of $S$ to make a given position become acceptable. However, they do not address the following issues treated in our paper: the discussion of no acceptability arbitrage, the characterization of when two risk measures coincide (which alllows to establish a link between multi-eligible-asset and single-eligible-asset risk measures), and the application of the method to a multi-currency setting.

Remark 3. The reader may recognize in $\rho_{\mathcal{A}, S}$ the infimal convolution (see Rockafellar (1970), Section 5) of the risk measure $\rho_{\mathfrak{A}, r}$ and of the risk measure given by the super-hedging with the assets in $\mathcal{S}$. The application of infimal convolution to risk measures has been first mentioned in Delbaen (2000), Section 4.3 ii).

Remark 4. Although, a priori, there is no rationale to restrict the chosen set $S$ of eligible assets to a strict subset of the universe of eligible assets, there may be practical reasons to do so. One of these could be the risk manager's lack of skills to operate safely in certain asset classes. In this sense, the set $S$ can be interpreted as the risk manager's potentially limited access to financial markets. In principle one could also consider allowing for non-traded assets to make an unacceptable financial position acceptable, e.g. an insurer may wish to use reinsurance to achieve acceptance. We do not explore this further and refer to Barrieu and El Karoui $(2002,2005)$ who also use infimal convolution. The following differentiates the two uses:

- theirs is applied in an economic framework of traded instruments as well as asymmetric over the counter trades: Sections 2.1.1 and 4.1 look for a "structure" (or "issue") $(F, \pi)$ which has to be optimal for agent A under a constraint set by agent $\mathrm{B}$ with both agents having a risk measure and total or partial access to a market,

- their paper does not deal with our "supervisory" approach where the decision maker is subject to conditions of acceptability by supervision,

- our paper is closer to the problem addressed in Dybvig (1992) of a unique agent dealing, in utility terms, with a non tradeable risk and with the market for elements in $\mathcal{S}$.

The authors thank Jean-Marc Eber for insisting on the necessity of an absence of "acceptability arbitrage", to avoid that any unacceptable position can be made acceptable with a portfolio long and short in eligible assets belonging to $S$ : 
Assumption $N A A(\mathcal{A}, \mathcal{S})$. No acceptability arbitrage: the space $\mathcal{M}_{0}(\mathcal{S})$ does not intersect the interior of $\mathcal{A}$.

Remark 1. Note that condition $N A L(\mathcal{A})$ of Section 1 implies condition $N A A(\mathcal{A}, \mathcal{S})$.

Remark 2. Using the representation result on coherent risk measures (see Prop. 4.1 in Artzner et al. (1999)) one can show that Assumption $N A A(\mathcal{A}, \mathcal{S})$ is equivalent to the existence of a test probability for $\rho_{\mathfrak{A}, r}$ which vanishes on each of the $s \in \mathcal{S}$ (see Delbaen (2000) for the case of a general state space $\Omega$ ).

The following result highlights the technical necessity of Assumption $N A A(\mathcal{A}, \mathcal{S})$.

Proposition 2. The number $\rho_{\mathfrak{A}, S}(X)$ is finite for all $X \in L^{0}$ if and only if Assumption $N A A(\mathcal{A}, \mathcal{S})$ holds. If Assumption $N A A(\mathcal{A}, \mathcal{S})$ does not hold, then $\rho_{\mathfrak{A}, S}(X)=$ $-\infty$ for all $X \in L^{0}$.

Proof. Take $r \in \mathcal{S}$, so that $r$ is strictly positive in all states of the world.

To prove the "if" part assume that $\rho_{\mathfrak{A}, S}(X)=-\infty$. Choose $\lambda>0$ such that $\lambda r-X$ is strictly positive in all states of the world. In particular, $\lambda r-X$ belongs to the interior of $\mathcal{A}$. Since, $\rho_{\mathcal{A}, S}(X)=-\infty$ we find a $Z \in \mathcal{M}(S)$ with $\pi(Z)=-\lambda$ and $X+Z \in \mathcal{A}$. It follows that $Y:=Z+\lambda r=(X+Z)+(\lambda r-X)$ belongs to the interior of $\mathcal{A}$. We conclude that $Y$ belongs to the intersection of $\mathcal{M}_{0}(S)$ and the interior of $\mathcal{A}$.

To prove the "only if part" assume there exists a $Y$ in the intersection of $\mathcal{M}_{0}(S)$ and the interior of $\mathcal{A}$. Choose $m>0$ small enough such that $Y-m r \in \mathcal{A}$. Then $Z_{\lambda}:=\lambda Y-\lambda m r \in \mathcal{A}$ for all $\lambda>0$. Since $\rho_{\mathcal{A}, s}(0) \leq \pi\left(Z_{\lambda}\right)=-\lambda m$ holds, we conclude that $\rho_{\mathfrak{A}, S}(0)=-\infty$.

To prove the second part of the proposition assume that $Y \in L^{0}$ is such that $\rho_{\mathcal{A}, S}(Y)=-\infty$. For each $m$ we find $Z_{m} \in \mathcal{M}(S)$ with $\pi\left(Z_{m}\right) \leq m$ and $Y+$ $Z_{m} \in \mathcal{A}$. For each $X \in L^{0}$ we find $\lambda>0$ such that $X+\lambda r>Y$.

We have $X+\lambda r+Z_{m}>Y+Z_{m}$ hence $X+\lambda r+Z_{m} \in \mathcal{A}$ and $\rho_{\mathcal{A}, s}(X+\lambda r) \leq$ $\pi\left(Z_{m}\right) \leq m$, hence $\rho_{\mathcal{A}, s}(X) \leq m-\lambda$. Since $m$ does not depend on $\lambda$ the conclusion that $\rho_{\mathfrak{A}, S}(X)=-\infty$ follows.

Remark 1. If Assumption $N A A(\mathcal{A}, \mathcal{S})$ does not hold, then the second part of Proposition 2 tells us that for any $X$ and any $m>0$ we can find a $Z \in \mathcal{M}(S)$ with $\pi(Z)=-m$ and $X+Z \in \mathcal{A}$. This means that any position $X$ can be made acceptable by "borrowing" the amount $m$ by going short the portfolio $Z$. This is what we have called "acceptability arbitrage".

Remark 2. Assume that $\mathcal{A}$ satisfies $N A A(\mathcal{A}, \mathcal{S})$. In very much the same way as in Artzner et al (1999) for the single-eligible-asset case, it is possible to show that $\rho_{\mathfrak{A}, S}: L^{0} \rightarrow \mathbb{R}$ satisfies the following properties

- monotonicity: for each $X$ and each $Y$, if $X \geq Y$ then $\rho_{\Re, S}(X) \leq \rho_{\Re, S}(Y)$,

- translation invariance: for each $Z \in \mathcal{M}(S)$ and each $X, \rho_{\mathcal{A}, S}(X+Z)=\rho_{\mathcal{A}, S}(X)-$ $\pi(Z)$, 
- positive homogeneity: if $\lambda \geq 0$ then for each $X, \rho_{\mathfrak{A}, S}(\lambda X)=\lambda \rho_{\mathfrak{A}, S}(X)$,

- subadditivity: for each $X$ and each $Y, \rho_{\mathfrak{A}, S}(X+Y) \leq \rho_{\mathfrak{A}, S}(X)+\rho_{\mathfrak{A}, S}(Y)$.

The translation invariance and monotonicity imply that $\rho_{\mathcal{A}, S}$ is globally Lipschitz continuous. The translation invariance stated above is the multi-eligible-asset version of the translation invariance in the single-eligible asset setting. Similarly to Artzner et al. (1999), Section 2.3, Definition 2.3, the four properties above can be used to define and develop the corresponding theory for multi-eligibleasset "coherent" risk measures without starting from an acceptance set (see Frittelli and Scandolo (2006), Proposition 3.6, and the independent work of Koch-Medina (2006)).

Under Assumption $N A A(\mathcal{A}, \mathcal{S})$ the "augmented" acceptance set $\mathcal{A}+\mathfrak{M}_{0}(S)$ is a coherent acceptance set:

Proposition 3. Let $\mathcal{A}$ be a coherent acceptance set and $\mathcal{S}$ a set of eligible assets. Then, $\mathcal{A}+\mathcal{M}_{0}(S)$ is a coherent acceptance set if and only if Assumption $N A A(\mathcal{A}, S)$ holds.

Proof. The set $\mathcal{A}+\mathcal{M}_{0}(S)$ is a convex homogeneous cone containing $L_{+}^{0}$. Hence we only need to prove that $\mathcal{A}+\mathcal{M}_{0}(S)$ does not intersect the interior of $L_{-}^{0}$ if and only if Assumption $N A A(\mathcal{A}, \mathcal{S})$ holds.

Assume first that $N A A(\mathcal{A}, \mathcal{S})$ holds. If for $X \in \mathcal{A}$ and $Z \in \mathcal{M}_{0}(\mathcal{S})$ we have that $X+Z$ belongs to $L_{--}^{0}$ we would find that $-Z$ belongs to the interior of $\mathcal{A}$, contradicting Assumption $N A A(\mathcal{A}, \mathcal{S})$.

To prove the converse assume that $\mathcal{A}+\mathcal{M}_{0}(S)$ does not intersect the interior of $L_{-}^{0}$. Let $r \in \mathcal{S}$ so that $r$ is strictly positive in all states of nature. If there exists $Z$ belonging to both $\mathcal{M}_{0}(S)$ and the interior of $\mathcal{A}$ we can find $\varepsilon>0$ such that $X:=Z-\varepsilon r \in \mathcal{A}$. It follows that $-\varepsilon r=X-Z$ belongs to both $\mathcal{A}+\mathcal{M}_{0}(S)$ and the interior of $L_{-}^{0}$, a contradiction.

Remark 1. Even if we had required acceptance cones to be closed, this would not guarantee that $\mathcal{A}+\mathcal{M}_{0}(S)$ would be closed, unless, for example, the cone $\mathcal{A}$ is polyhedral (see Rockafellar (1970), Section 19).

Remark 2. Assumption $N A A(\mathcal{A}, S)$ does not imply that $\mathcal{A}+\mathcal{M}_{0}(S)$ is closed if $\mathcal{A}$ is closed. Note however that the assumption of non-acceptability of leveraged positions introduced in Section 1 (Assumption $\operatorname{NAL}(\mathcal{A})$ ) ensures that $\mathcal{M}_{0}(\mathcal{S}) \cap$ $\overline{\mathcal{A}}=\{0\}$, which implies that $\overline{\mathcal{A}}+\mathcal{M}_{0}(S)$ is closed.

We next characterize when two measures $\rho_{\mathcal{A}, S}$ and $\rho_{\mathcal{B}, \mathcal{T}}$ are equal. But first we prove a useful technical lemma.

Lemma. If $\mathcal{A}$ is a coherent acceptance set and $\mathcal{S}$ is a set of eligible assets satisfying Assumption $\operatorname{NAA}(\mathcal{A}, \mathcal{S})$ we have:

$$
\rho_{\mathfrak{A}, S}(X) \leq 0 \text { if and only if } X \text { belongs to the closure of } \mathcal{A}+\mathcal{M}_{0}(\mathcal{S}) .
$$


In addition,

$$
\rho_{\mathfrak{A}, S}=\rho_{\overline{\mathfrak{A}}, \mathcal{S}}=\rho_{\mathfrak{A}+\mathfrak{M}_{0}(S), S}=\rho_{\overline{\mathcal{A}+\mathscr{M}_{0}(S)}, S} .
$$

Proof. We only prove the first part of the lemma. Assume that $X$ does not belong to the closure of $\mathcal{A}+\mathcal{M}_{0}(S)$ and set $m:=\rho_{\mathcal{A}, S}(X)$. Take now $m_{k} \backslash m$ and $Z_{k} \in \mathcal{M}(S)$ such that $\pi\left(Z_{k}\right)=m_{k}$ and $X+Z_{k} \in \mathcal{A}$. Then, $Y:=m_{k} r-Z_{k}$ belongs to $\mathcal{M}_{0}(S)$ and

$$
X+m_{k} r-Y=X+Z_{k} \in \mathcal{A} .
$$

It follows that $X+m_{k} r \in \mathcal{A}+\mathcal{M}_{0}(S)$. Passing to the limit we find: $X+m r$ belongs to the closure of $\mathcal{A}+\mathcal{M}_{0}(S)$.

If $m \leq 0$, then $X+m r \leq X$ and, therefore, $X$ belongs to the closure of $\mathcal{A}+$ $\mathcal{M}_{0}(S)$, since this set is itself a coherent acceptance set. This contradicts the choice of $X$ and $m>0$ must thus hold. This proves that $\rho_{\mathcal{A}, S}(X) \leq 0$ implies that $X$ belongs to the closure of $\mathcal{A}+\mathcal{M}_{0}(S)$.

To prove the converse implication take $X$ in the closure of $\mathcal{A}+\mathcal{M}_{0}(S)$. Then for each $\varepsilon>0$ we find $Y+Z \in \mathcal{A}+\mathcal{M}_{0}(S)$ such that $Y+Z \leq X+\varepsilon r$. It follows that $Y \leq X-Z+\varepsilon r$ and hence $X-Z+\varepsilon r \in \mathcal{A}$. Since $\pi(-Z+\varepsilon r)=\varepsilon$ we find that $\rho_{\mathfrak{A}, S}(X) \leq \varepsilon$ which implies that $\rho_{\mathfrak{A}, S}(X) \leq 0$ since $\varepsilon>0$ was arbitrary.

Remark 1. Using the lemma above one can prove that for each $X$ we find a $Z \in \mathcal{M}(S)$ with $\pi(Z)=\rho_{\mathfrak{A}, S}(X)$ and $X+Z \in \mathcal{A}$ if and only if $\mathcal{A}+\mathcal{M}_{0}(S)$ is closed. This is in general not the case. However, if Assumption $\operatorname{NAL}(\mathcal{A})$ holds we do have that $\overline{\mathcal{A}}+\mathcal{M}_{0}(S)$ is closed and as a result, in this case, we have that there always exists $Z \in \mathcal{M}(S)$ with $\pi(Z)=\rho_{\mathcal{A}, S}(X)$ and $X+Z \in \overline{\mathcal{A}}$.

Remark 2. As in Artzner et al. (1999), Section 2.3, the risk measure $\rho_{\mathcal{A}, S}$ is said to be relevant whenever for any non-zero $X \in L_{-}^{0}$ we have $\rho_{\mathcal{A}, S}(X)>0$. Using the lemma above one can prove that $\rho_{\mathcal{A}, S}$ is relevant if and only if $\mathcal{A}+\mathcal{M}_{0}(S)$ intersects $L_{-}^{0}$ only at $\{0\}$.

We can now state and prove a key result of this paper.

Theorem. Let $\mathcal{S}$ and $\mathcal{T}$ be sets of eligible assets. Let $\mathcal{A}$ and $\mathcal{B}$ be coherent acceptance sets satisfying assumptions $N A A(\mathcal{A}, \mathcal{S})$ and $N A A(\mathcal{B}, \mathcal{T})$, respectively. Assume further that $S$ and $\mathcal{T}$ have non-empty intersection. Then, $\rho_{\mathcal{A}, \mathcal{S}}=\rho_{\mathcal{B}, \mathcal{T}}$ if and only if the closures of the sets $\mathcal{A}+\mathcal{M}_{0}(S)$ and $\mathcal{B}+\mathcal{M}_{0}(\mathcal{T})$ coincide.

Proof. Assume that the closures of $\mathcal{A}+\mathcal{M}_{0}(S)$ and $\mathcal{B}+\mathcal{M}_{0}(\mathcal{T})$ coincide and take $r \in \mathcal{S} \cap \mathcal{T}$. For any $X$ take $m>\rho_{\mathcal{A}, S}(X)$ and $Z \in \mathcal{M}(S)$ with $\pi(Z)=m$ and $X+Z \in \mathcal{A}$. Then,

$$
X+m r+(Z-m r) \in \mathcal{A}
$$

Since $m r-Z \in \mathcal{M}_{0}(S)$ we see that $X+m r$ belongs to $\mathcal{A}+\mathcal{M}_{0}(S)$ and, hence, to the closure of $\mathcal{B}+\mathcal{M}_{0}(\mathcal{T})$. It follows from the lemma above that 


$$
\rho_{\mathcal{B}, \mathcal{T}}(X)=\rho_{\bar{B}+\mathscr{M}_{0}(\mathcal{T}), \mathcal{T}}(X)<m .
$$

Consequently, $\rho_{\mathcal{B}, \mathcal{T}} \leq \rho_{\mathfrak{A}, \mathcal{S}}$. Since the argument is symmetric in $\mathcal{A}$ and $\mathcal{B}$ we get $\rho_{\mathcal{B}, \mathcal{T}}=\rho_{\mathfrak{A}, \mathcal{S}}$.

Assume now that $\rho_{\mathcal{B}, T}=\rho_{\mathfrak{A}, S}$. From the lemma above it immediately follows that the closures of $\mathcal{A}+\mathcal{M}_{0}(\mathcal{S})$ and $\mathcal{B}+\mathcal{M}_{0}(\mathcal{T})$ coincide.

The result above tells us that the risk measure is essentially determined by acceptance up to hedging with eligible assets at zero initial cost. The following result is an immediate consequence of the Theorem.

Corollary. Under $N A A(\mathcal{A}, \mathcal{S})$ the risk measure $\rho_{\mathfrak{A}, \mathcal{S}}$ is equal for any one say $t$ of the eligible assets in $\mathcal{S}$ to the measure $\rho_{\mathfrak{A}+\mathcal{M}_{0}(S), t}$.

It follows that the allowance of more than one eligible asset amounts to "augmenting" the original acceptance set $\mathcal{A}$ to $\mathcal{A}+\mathcal{M}_{0}(S)$ and choosing any of the original eligible assets as the single eligible asset.

\section{COHERENT RISK MEASURES IN A MULTI-CURRENCY SETTING: ACCOUNTING}

Two issues concerning risk measurement in a multi-currency setting provided the original motivation for the work presented in this paper.

The first issue had to do with capital efficiency. The example in the Appendix shows that using either the domestic or the foreign risk-free asset as the sole eligible asset will generally lead to an inefficient use of capital in the sense that the risk measure will not correspond to the cheapest way to make a position acceptable. This has lead some industry participants to leave the single-eligibleasset setting and allow investing in the domestic and foreign "risk-free assets" when measuring risk. That lead us to consider multi-eligible-asset risk measures which, as we showed in Section 2, turn out to possess reasonable (coherence) properties.

The second issue, which is the focus of this section, had to do with the question of what happens when we change the currency we account in. A natural requirement is that if a company were to calculate risk using a domestic and a foreign risk measure, these measures should be compatible in the sense that it should not matter whether we first calculate the capital requirement in the foreign currency and then translate it into domestic currency at the spot rate (exchange rate at time $t=0$ ), or perform all calculations in domestic currency in the first place. This requirement is natural because changing the unit of account is essentially a different way to quote prices and should by itself have no impact on whether a financial position is accepted or not. This issue is related to the characterization of when two risk measures are equal, which was addressed in Proposition 1 in Section 1 for the single-eligible-asset setting and in the Theorem in Section 2 for the multi-eligible-asset setting. 
We consider a two currencies setting, where $e_{t}$ denotes the amount of units of foreign currency which one unit of domestic currency buys at time $t \in\{0,1\}$. Thus $e_{0}$ is a strictly positive number and $e_{1}$ is an $\mathcal{F}$-measurable random variable which is strictly positive in all states of nature.

In the previous sections we fixed the currency in which prices and future values of financial positions were expressed. We now need to differentiate and hence, if we are given a set $\mathcal{A}^{d}$ of future values of financial positions quoted in domestic currency the future values of these financial positions expressed in foreign currency are the elements of the set $e_{1} \mathcal{A}^{d}$.

Definition 3. Compatibility of risk measures in various currencies: Let $\mathcal{A}^{d}$ and $\mathcal{B}^{f}$ be coherent acceptance sets, expressed in domestic and foreign currency, respectively. In addition, let $\mathcal{S}^{d}$ and $\mathcal{T}^{f}$ be sets of eligible assets expressed in domestic and foreign currency, respectively. The "domestic" risk measure $\rho_{\mathbb{A}^{d}}, S^{d}$ and the "foreign" risk measure $\rho_{B^{f}, T^{f}}$ are said to be compatible whenever the equality

$$
e_{0} \rho_{\mathfrak{A}^{d}, S^{d}}(X)=\rho_{\mathcal{B}^{f}, \mathcal{T}^{f}}\left(e_{1} X\right)
$$

holds for all future values $X$ of financial positions denominated in domestic currency.

Remark. The compatibility of $\rho_{\mathfrak{A}^{d}, S^{d}}$ and $\rho_{\mathcal{B}^{f}, \mathcal{T}^{f}}$ thus means that they are in fact domestic, respectively, foreign versions of the same "accounting-unit-free" risk measure. In effect, we have chosen to do this section in the context of changing the currency in which we account (via domestic or foreign cash), but similar ideas can be developed to study any change of numéraire without a change of investment vehicle.

We first look at compatibility in the single-eligible-asset context. Choose a domestic coherent acceptance set $\mathcal{A}^{d}$ and a foreign coherent acceptance set $\mathcal{B}^{f}$. Choose also a domestic eligible asset $r^{d}$ and a foreign eligible asset $s^{f}$. Compatibility is easily shown to be equival ent to the equality $\rho_{\mathfrak{A}^{d}, r^{d}}=\rho_{\frac{1}{e_{1}} \mathfrak{B}^{f}, \frac{e_{0}}{e_{1}} f^{f}}$.

An intuitive case is the one where $r^{d}$ and $s^{f}$ are the expressions in the different currencies of the same financial asset, which translates into the equality $e_{1} r^{d}=e_{0} s^{f}$. In this case compatibility is reached if and only if $e_{1} \mathcal{A}^{d}$ and $\mathcal{B}^{f}$ have the same closure.

Note that if for instance $r^{d}$ and $s^{f}$ are the (one-period) "risk-free" eligible assets in the domestic and foreign currency respectively, under a genuinely random exchange rate, we are facing an instance of the opposite case where the equality $e_{1} r^{d}-e_{0} s^{f}=0$ is not satisfied. We examine this case now with the help of Proposition 1-b:

Proposition 4. If either $N A L\left(\mathcal{A}^{d}\right)$ or $N A L\left(\mathcal{B}^{f}\right)$ holds then compatibility of $\rho_{\mathscr{A}^{d}, r^{d}}$ and $\rho_{\mathcal{B}^{f}, s^{f}}$ is impossible when $e_{1} r^{d} \neq e_{0} s^{f}$.

Remark 1. The fact that $\rho_{\mathfrak{A}^{d}, r^{d}}$ and $\rho_{\mathcal{B}^{f}, s^{f}}$ are not compatible in general had been noticed in Artzner et al. (1999), Remark 2.5, and it is illustrated in the paper's Appendix. 
Remark 2. Assume that the domestic and foreign coherent acceptance sets $\mathcal{A}^{d}$ and $\mathcal{B}^{f}$ are both defined in their respective currencies in terms of the same mathematical functional e.g. TailVaR $R_{\alpha}$ for a given level $\alpha \in(0,1)$ :

$$
\mathcal{A}^{d}=\left\{X \in L^{0} \mid \operatorname{TailVaR}_{\alpha}(X) \leq 0\right\} \text { and } \mathcal{B}^{f}=\left\{Y \in L^{0} \mid \operatorname{TailVaR}_{\alpha}(Y) \leq 0\right\} .
$$

Since the closed sets $e_{1} \mathscr{A}^{d}$ and $\mathcal{B}^{f}=\mathscr{A}^{d}$ will generally not coincide if $e_{1}$ is genuinely random, the associated risk measures are generally not compatible. This example warns of a potential pitfall when defining acceptance through a specific functional: the functional form is valid for a given reference currency (or unit of account) and is usually lost when changing to a different one. We direct the reader to the second numerical example in the Appendix.

Remark 3. Note moreover that the definition, after a currency choice, of a TailVaR acceptance set in Remark 2 is different from the one given in Artzner et al. (1999), and most of the subsequent literature. There, probably under the influence of the representation theorem for coherent risk measures (Proposition 4.1), the acceptance set corresponding to the functional TailVaR $R_{\alpha}$ in a given currency had been defined as $\left\{X \mid \operatorname{TailVar}_{\alpha}\left(\frac{X}{r}\right) \leq 0\right\}$, where $r$ was the eligible asset (Definition 5.1).

This definition was unfortunate since the acceptance set should not be depend on the choice of the eligible asset (see Remark 1 in Section 1 on the conceptual primacy of the acceptance set). Only when measuring risk does the eligible asset come into play. It is the "yardstick" we use to measure how much additional capital we need to invest today to ensure acceptability.

In the multi-eligible-asset case we have the following consequence of the corollary to the theorem in Section 2.

Proposition 5. Let $\mathcal{S}^{d}$ and $\mathcal{T}^{f}$ be sets of eligible assets expressed in domestic and foreign currency, respectively. In addition, let $\mathcal{A}^{d}$ be domestic and $\mathcal{B}^{f}$ foreign coherent acceptance sets satisfying $N A A\left(\mathscr{A}^{d}, \mathcal{S}^{d}\right)$ and $N A A\left(\mathcal{B}^{f}, \mathcal{T}^{f}\right)$, respectively. Finally, assume that the intersection of $S^{d}$ and $\frac{e_{0}}{e_{1}} \mathcal{T}^{f}$ is not empty. Then, the measures $\rho_{\mathcal{A}^{d}, S^{d}}$ and $\rho_{\mathcal{B}^{f}, \mathcal{T}^{f}}$ are compatible if and only if the closures of the sets $e_{1}\left(\mathcal{A}^{d}+\mathcal{M}_{0}\left(S^{d}\right)\right)$ and $\mathcal{B}^{f}+\mathcal{M}_{0}\left(\mathcal{T}^{f}\right)$ coincide.

Proof. It is possible to find $r^{d} \in \mathcal{S}^{d}, s^{f} \in \mathcal{T}^{d}$ with $e_{1} r^{d}=e_{0} s^{f}$. We may identifiy $\rho_{\mathfrak{A}^{d}, S^{d}}$ and $\rho_{\mathcal{B}^{f}, \mathcal{T}^{f}}$ to $\rho_{\mathfrak{A}^{d}+\mathfrak{M}_{0}\left(S^{d}\right), r^{d}}$ and $\rho_{\mathcal{B}^{f}+\mathfrak{M}_{0}\left(\mathcal{T}^{f}\right), s^{f}}$ respectively. These two measures are compatible hence the conclusion of the single-eligible-asset case leads to the stated result. 


\section{APPENDIX}

\section{An inefficient use of capital}

The following example illustrates an inefficient use of capital in presence of two currencies.

For one period of uncertainty with three equally weighted states of nature suppose the current exchange rate $e_{0}$ being 1 and the future rate $e_{1}$ being $\left(\frac{1}{2}, 1,2\right)$ in the respective three states. Assume zero interest rate in either currency and consider the eligible assets $r^{d}=s^{f}=1$ (the reader can check that the condition $e_{1} r^{d}=e_{0} s^{f}$ of Proposition 4 is not fulfilled). For simplicity use as, admittedly extreme, acceptance sets $\mathscr{A}^{d}$ and $\mathcal{B}^{f}$ the positive orthant $\mathbb{R}_{+}^{3}$ (hence $e_{1} \mathscr{A}^{d}=\mathcal{B}^{f}$ ) which means that both $\rho_{\mathfrak{A}^{d}, r^{d}}$ and $\rho_{\mathcal{B}^{f}, s^{f}}$ are given by the "worst case scenario" $\rho((a, b, c))=-\min (a, b, c)$.

Consider a "domestic" random future value $X=(-16,1,-7)$. Making it acceptable using domestic currency requires 16 units at a domestic cost of 16, while $b$ units of current foreign currency will provide acceptance as long as $X+\left(2 b, b, \frac{b}{2}\right)$ is in the positive orthant $\mathcal{A}^{d}$, that is $\frac{b}{2}-7 \geq 0$, at a (different) domestic cost of 14. It is more efficient to use a bit of foreign currency to offset the loss should the first state obtain and domestic currency to offset the loss in the third state: a 2 by 3 linear program shows that 4 units of domestic currency together with 6 units of foreign currency, at a total cost of 10, suffice for acceptability. One can say that given the "global" character of $X$ it is not surprising that a "buffer" made of a mix of currencies is cheaper than a singlecurrency buffer.

\section{Incompatibility of TailVaR based risk measures denominated in different currencies}

The following example illustrates why TailVaR based risk measures denominated in different currencies fail to be compatible.

Let $\Omega=\left\{\omega_{1}, \ldots, \omega_{100}\right\}$ describe the states of nature, each having probability $\frac{1}{100}$, and $e_{1}: \Omega \rightarrow(0, \infty)$ be the non-constant rate at which one domestic currency unit is exchanged into foreign currency at time 1 . Take $\alpha=2 \%$, then for any $Y: \Omega \rightarrow \mathbb{R}$ with $Y\left(\omega_{1}\right) \geq Y\left(\omega_{2}\right) \geq \ldots \geq Y\left(\omega_{98}\right)>\max \left\{Y\left(\omega_{99}\right), Y\left(\omega_{100}\right)\right\}$ we have

$$
\operatorname{TailVaR}_{\alpha}(Y)=-\frac{Y\left(\omega_{99}\right)+Y\left(\omega_{100}\right)}{2} .
$$

Assume without loss of generality that $e_{1}\left(\omega_{1}\right) \geq e_{1}\left(\omega_{2}\right) \geq \ldots \geq e_{1}\left(\omega_{98}\right) \geq e_{1}\left(\omega_{99}\right)>$ $e_{1}\left(\omega_{100}\right)$ holds. Define $X: \Omega \rightarrow \mathbb{R}$ by $X\left(\omega_{j}\right)=1$ for $1 \leq j \leq 98$ and $X\left(\omega_{99}\right)=$ $-X\left(\omega_{100}\right)=-0.5$. Then, $e_{1}\left(\omega_{99}\right) X\left(\omega_{99}\right)+e_{1}\left(\omega_{100}\right) X\left(\omega_{100}\right)<0$, TailVaR $R_{\alpha}(X)=0$ and TailVaR $R_{\alpha}\left(e_{1} X\right)>0$. As a result $e_{1} \mathscr{A}^{d} \neq \mathcal{B}^{f}$ where $\mathscr{A}^{d}=\left\{X \in L^{0} \mid \operatorname{TailVa}_{\alpha}(X) \leq 0\right\}$ and $\mathcal{B}^{f}=\left\{Y \in L^{0} \mid \operatorname{TailVaR}_{\alpha}(Y) \leq 0\right\}$. From this and Proposition 4, it follows that $\rho_{\mathfrak{A}^{d}, r^{d}}$ and $\rho_{\mathcal{B}^{f}, s^{f}}$ cannot be compatible for any choice of domestic and foreign eligible assets $r^{d}$ and $s^{f}$. 


\section{REFERENCES}

Artzner, Ph., Delbaen, F., Eber, J.-M. and Heath, D. (1997) Thinking coherently, Risk 10, 68-71.

Artzner, Ph., Delbaen, F., Eber, J.-M. and Heath, D. (1999) Coherent Risk Measures, Mathematical Finance 9, 203-228.

Artzner, Ph., Delbaen, F., Eber, J.-M., Heath, D. and Ku, H. (2007) Coherent multiperiod risk-adjusted values and Bellman's principle, Annals of Operations Research 152, 5-22.

Artzner, Ph., Delbaen, F., Koch-Medina, P. (2005) Risk Measures and Efficient Use of Capital, Proceedings 15th International AFIR Colloquium, Zürich, September 6-9.

Aumann, R. and Serrano, R. (2006) An Economic Index of Riskiness, Working paper, Department of Economics, Brown University.

Barrieu, P. and El Karoui, N. (2002) Optimal Risk Transfer, Working Paper, LSE and Ecole Polytechnique.

BARrieu, P. and El Karoui, N. (2005) Inf-convolution of risk measures and optimal risk transfer, Finance and Stochastics 9, 269-298.

Committee of European Insurance and Occupational Pensions Supervisors (2007) Advice to the European Commission in the Framework of the Sovency II Project, March.

Delbaen, F. (2000) Coherent Risk Measures, Lectures Scuola Normale Superiore, Pisa.

Dybvig, Ph. (1992) Hedging Nontraded Wealth: When is there Separation of Hedging and Investment?, Options: Recent Advances in Theory and Practice 2 (Hodges, S.D., ed.), Manchester University Press.

Filipovic, D. (2008) Optimal Numeraires for Risk Measures, Mathematical Finance 18, 333-336, earlier version December 2006.

Föllmer, H. and Schied A. (2004) Stochastic Finance, 2nd ed., de Gruyter, Berlin.

Frittelli, M. and Scandolo, G. (2006) Risk measures and capital requirements for processes, Mathematical Finance 16, 589-612.

Koch-Medina, P. (2006) Generalized coherent risk measures, Unpublished manuscript.

Rockafellar, T. (1970) Convex Analysis, Princeton University Press, Princeton, NJ.

Stickney, C., Weil, R. and Davidson, S. (1991) Financial Accounting, 6th ed., Harcourt Brace Jovanovich, Orlando, FA.

PHILIPPE ARTZNER

Institut de Recherche Mathématique Avancée,

Université de Strasbourg et CNRS et Laboratoire de Recherches en Gestion, FR 67084 Strasbourg

France

E-Mail: artzner@math.u-strasbg.fr

Freddy Delbaen

Departement für Mathematik

Eidgenössische Technische Hochschule,

ETH-Zentrum, CH 8092 Zürich

Schweiz

E-Mail:delbaen@math.ethz.ch

Pablo Koch-Medina

Swiss Reinsurance Company,

Mythenquai 50/60, CH 8022 Zürich

Schweiz

E-Mail:Pablo_KochMedina@swissre.com 